\title{
Development and characterization of a brain tumor mimicking fluorescence phantom
}

\author{
Neda Haj-Hosseini, Benjamin Kistler and Karin Wårdell
}

\section{Linköping University Post Print}

\section{Tweet}

N.B.: When citing this work, cite the original article.

Original Publication:

Neda Haj-Hosseini, Benjamin Kistler and Karin Wårdell, Development and characterization of a brain tumor mimicking fluorescence phantom, 2014, Proceedings of SPIE, the International Society for Optical Engineering, (8945), 894505, 1-6.

http://dx.doi.org/10.1117/12.2039861

Copyright: Society of Photo-optical Instrumentation Engineers (SPIE)

http://spie.org/

Postprint available at: Linköping University Electronic Press

http://urn.kb.se/resolve?urn=urn:nbn:se:liu:diva-98989 


\title{
Development and Characterization of a Brain Tumor Mimicking Fluorescence Phantom
}

\author{
Neda Haj-Hosseini*a ${ }^{a}$ Benjamin Kistler ${ }^{\mathrm{a}, \mathrm{b}}$, Karin Wårdell ${ }^{\mathrm{a}}$ \\ ${ }^{a}$ Department of Biomedical Engineering, Linköping University, Linköping, Sweden, \\ ${ }^{\mathrm{b}}$ Institute of Medical and Analytical Technologies, University of Applied Sciences Northwestern Switzerland, \\ Switzerland
}

\begin{abstract}
Fluorescence guidance using 5-aminolevulinic acid (5-ALA) for brain tumor resection is a recent technique applied to the highly malignant brain tumors. Five-ALA accumulates as protoporphyrin IX fluorophore in the tumor cells in different concentrations depending on the tumor environment and cell properties. Our group has developed a fluorescence spectroscopy system used with a hand-held probe intra-operatively. The system has shown improvement of fluorescence detection and allows quantification that preliminarily correlates with tumor malignancy grade during surgery. However, quantification of fluorescence is affected by several factors including the initial fluorophore concentration, photobleaching due to operating lamps and attenuation from the blood. Accordingly, an optical phantom was developed to enable controlled fluorescence measurements and evaluation of the system outside of the surgical procedure. The phantom mimicked the optical properties of glioma at the specific fluorescence excitation wavelength when different concentrations of the fluorophore were included in the phantom. To allow evaluation of photobleaching, kinetics of fluorophore molecules in the phantom was restricted by solidifying the phantoms. Moreover, a model for tissue autofluorescence was added. The fluorescence intensity's correlation with fluorophore concentration in addition to the photobleaching properties were investigated in the phantoms and were compared to the clinical data measured on the brain tumor.
\end{abstract}

Keywords: Protoporphyrine (PpIX), 5-aminolevulinic acid (5-ALA), autofluorescence, photobleaching, fluorescence guided resection, spectroscopy

\section{INTRODUCTION}

Fluorescence guidance using 5-aminolevulinic acid photosensitizer for resection of highly malignant brain tumors is an optical imaging method which has recently entered the clinical routine. Five-ALA, administered orally prior to surgery, accumulates in the tumor cells as protoporphyrine IX (PpIX) as a result of tumor's broken blood brain barrier and altered enzyme levels [1,2]. The conventional guidance is performed through modified surgical microscopes [3], as a technical alternative for detection of fluorescence, hand-held fiber-optical probes have been suggested for their higher detection sensitivity, fluorescence quantification possibilities and navigational features [4-8].

In the attempt to quantify the fluorescence signals, methods have been proposed which combine other optical modalities, apply Monte Carlo simulations or use the tissue autofluorescence to calculate the amount of PpIX concentration in the tissue [9-12]. The quantification methods which are based on fluorescence signals alone have the advantage of offering more practical and real-time measurements feasible during surgery. Detection of PpIX fluorescence has the main challenges of being highly affected by blood and photobleaching when exposed to surgical lamps or blue excitation light. These factors when not eliminated significantly affect the diagnosis which is based on the intensity of the fluorescence, either visually observed or measured with a fiber-optical probe. To validate any of the quantification methods it is essential to apply optical phantoms which in addition to the controlled photosensitizer concentrations implement the tissue fluorescence (autofluorescence) and photobleaching properties. In this paper, we present a preliminary phantom model for PpIX-enhanced brain tumor in which photobleaching properties of tissue are considered and compared to the data obtained from brain tumors.

*neda.haj.hosseini@liu.se; phone 4610103 2488; fax 4613 101902; imt.liu.se

Design and Performance Validation of Phantoms Used in Conjunction with Optical Measurement of Tissue VI, edited by Robert J. Nordstrom, Jean-Pierre Bouchard, David W. Allen, Proc. of SPIE Vol. 8945, 894505

(C) 2014 SPIE · CCC code: 1605-7422/14/\$18 · doi: 10.1117/12.2039861 


\section{MATERIAL AND METHODS}

\subsection{Optical properties of the brain tumor}

Optical properties of the brain tumor have been measured in several literature [13-15] according to the cited references are included in Table 1. Different malignancy grades demonstrate different optical properties from each other and from the normal brain tissue $[14,15]$. The data provided by Gebhart [13] was adapted for the phantoms.

Table 1. Optical properties of glioma and normal brain tissue at $405 \mathrm{~nm}$

\begin{tabular}{lcccc} 
& $\boldsymbol{\mu}_{\mathbf{a}}\left[\mathbf{m m}^{-1}\right]$ & $\boldsymbol{\mu}_{\mathbf{s}}\left[\mathbf{m m}^{-1}\right]$ & $\mathbf{g}$ & Reference \\
\hline Glioma & 1.3 & 26 & 0.85 & {$[13]$} \\
\hline Gray matter & 0.2 & 15 & 0.86 & {$[15]$} \\
\hline White matter & 0.3 & 40 & 0.75 & {$[15]$} \\
\hline
\end{tabular}

\subsection{Phantom composition}

The phantoms were prepared to have the optical properties of the high grade brain tumor tissue [13]; i.e., added scattering $\left(\mu_{\mathrm{s}}=26 \mathrm{~mm}^{-1}\right)$, absorption $\left(\mu_{\mathrm{a}}=4 \mathrm{~mm}^{-1}\right)$ and autofluorescence. Intralipid $20 \%$ (Fresenius Kabi, Uppsala, Sweden) and black ink was used to induce scattering and absorption properties, respectively. A yellow fluorescent objective slide (Chroma Technology Corp, Bellow Falls, VT, USA) was used to produce autofluorescence. Protoporphyrin IX Disodium Salt (MP Biomedicals, France) dissolved in dimethyl sulfoxide (DMSO, Thermo Fisher Scientific Inc., France) was used in concentrations of 2, 6, 10, 14 and $18 \mathrm{mgl}^{-1}$. These concentrations were chosen according to the PpIX concentration in the glioma samples [16]. To obtain a solid platform for photobleaching measurements, agarose gel $0.6 \%\left[\mathrm{~g} \mathrm{ml}^{-1}\right]$ was used to add identical mechanical properties of brain to the phantom (Young's modulus, $\mathrm{E}=10 \mathrm{kPa}$ ) $[17,18]$.

\subsection{Spectral collimated transmission}

The scattering and absorption properties of the phantom ingredients were measured using a spectral collimated transmission setup (SCT) [19]. For obtaining the broad spectrum a white light (AvaLight-Hal-S, Avantes, Apeldoorn, Netherlands) and for obtaining optical properties at $405 \mathrm{~nm}$, a blue laser light was used. Attenuation of the samples were measured and calculated using Beer-Lambert Law assuming intralipid as a scattering dominant medium and ink as an absorption dominant medium. Measurements were performed on three samples at each concentration.

\subsection{Fluorescence spectroscopy instrument}

The phantom was evaluated using a fluorescence spectroscopy system with a fiber-optical hand-held probe with which guided brain tumor resection is performed [4]. The probe used in the clinical measurements had a central fiber for excitation $\left(\varnothing_{\text {core }}=600 \mu \mathrm{m}\right.$, numerical aperture $\left.=0.37\right)$ and surrounding fibers for fluorescence collection $\left(\varnothing_{\text {core }}=200 \mu \mathrm{m}\right.$, numerical aperture $\left.=0.22\right)$. An identical probe was used on phantoms to avoid chemical contamination of the clinical probes. The probe composed of a central collecting fiber and six surrounding fibers for excitation $\left(\varnothing_{\text {core }}=400 \mu \mathrm{m}\right.$, numerical aperture $=0.22$ ). The laser and spectrometer were synchronized meaning that the integration time of the spectrometer was equal to the pulse width of laser light delivery.

\subsection{Experimental settings}

A multi-well sample holder was used to hold phantoms $(\varnothing=6.6 \mathrm{~mm})$. Each well was filled with $60 \mu \mathrm{l}$ of the phantom solution which gave a $1.7 \mathrm{~mm}$ thickness to the phantoms. The surrounding wells of each phantom well were filled with food color to avoid exposure of the phantoms to the excitation light (Figure 1). The fiber-optical probe was fixated on a micro-positioner for exact positioning above each phantom. The yellow fluorescent objective slide was placed behind the phantom sample holder as a model for tissue autofluorescence. A black plate was positioned behind the fluorescent slide to avoid reflection from the background surface.

Phantoms were prepared to have PpIX concentration of 2, 6, 10, 14 and $18 \mathrm{mgl}^{-1}$. Experiments on the phantoms were performed using excitation powers of $5,7.5$ and $10 \mathrm{~mW}$ with integration time of $0.2,0.4,0.6$ and $1 \mathrm{~s}$ to allow evaluation of the quantification method using different power settings. All the measurements were performed using continuous 
excitation. Signals with a weak autofluorescence below the system noise (20 photon counts [a.u.]) were excluded. Quantification was done by dividing the intensity at $634 \mathrm{~nm}$ by the maximum autofluorescence intensity (Figure $4 \mathrm{~b}$ ). The presented data from brain tumor correspond to an ALA dose of $5 \mathrm{mg} / \mathrm{kg}$ bodyweight [5]. The clinical measurements were approved by the local ethics committee (No: M139-07) with received written consent from the patients.

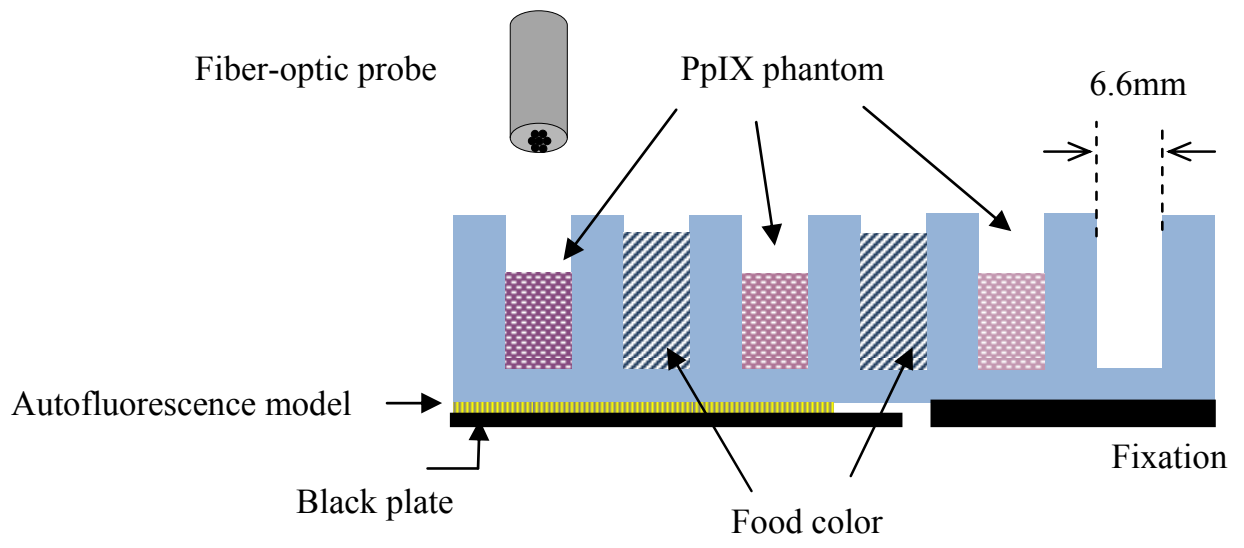

Figure 1: Experimental setup for the phantoms

\subsection{Analysis}

MATLAB version R2013a (The MathWorks, Inc., Natick, USA) was used to analyze the signals. Minitab software (Minitab, Inc, UK) was used for statistical analysis. Photobleaching data presented in Figure 5 are the curve-fits of the measurement points [20]. Pearson's linear correlation was used for correlation analysis between two groups (Figure 4) and Kruskal-Wallis test was used to analyse significant difference among groups presented in Figure 4b.

\section{RESULTS}

\subsection{Phantom composition}

The amount of the phantom ingredients and the induced properties are summarized in Table 2 . The volume concentration, $\rho$, of ink and intralipid may be calculated from Equations 1 and 2. $\mu_{a \text { (ink) }}$ is the absorption coefficient of ink $\left[\mathrm{mm}^{-1}\right]$ and $\mu_{s(i)}$ is the scattering coefficient of intralipid $\left[\mathrm{mm}^{-1}\right]$ at $405 \mathrm{~nm}$ measured using SCT setup. Absorption and fluorescence emission bands of PpIX are illustrated in Figure 2. Absorption coefficient of PpIX at $405 \mathrm{~nm}$, $\mu_{a(P p I X)}\left[\mathrm{mm}^{-1}\right]$, was a linear function of PpIX concentration, $c_{P p I X}\left[\mathrm{mg} \mathrm{l}^{-1}\right]$ (Equation 3).

$$
\begin{gathered}
\mu_{a(\text { ink })}=146.6 \rho_{i n k} \\
\mu_{s(i l)}=222.1 \rho_{i l} \\
\mu_{a(P p I X)}=0.3 c_{P p I X}
\end{gathered}
$$

Table 2. Amount of the ingredients used in the phantom and the induced properties

\begin{tabular}{lll} 
Substance & \multicolumn{1}{c}{ Amount } & property \\
\hline Intralipid $20 \%$ & $11.8 \%$ in total volume & $\mu_{\mathrm{s}}=26 \mathrm{~mm}^{-1}$ \\
\hline Black ink & $1.1 \%$ in total volume & $\mu_{\mathrm{a}}=1.3 \mathrm{~mm}^{-1}$ \\
\hline Agarose gel & $0.6 \% \mathrm{~g} \mathrm{ml}^{-1}$ & $\mathrm{E}=10 \mathrm{kPa}$
\end{tabular}




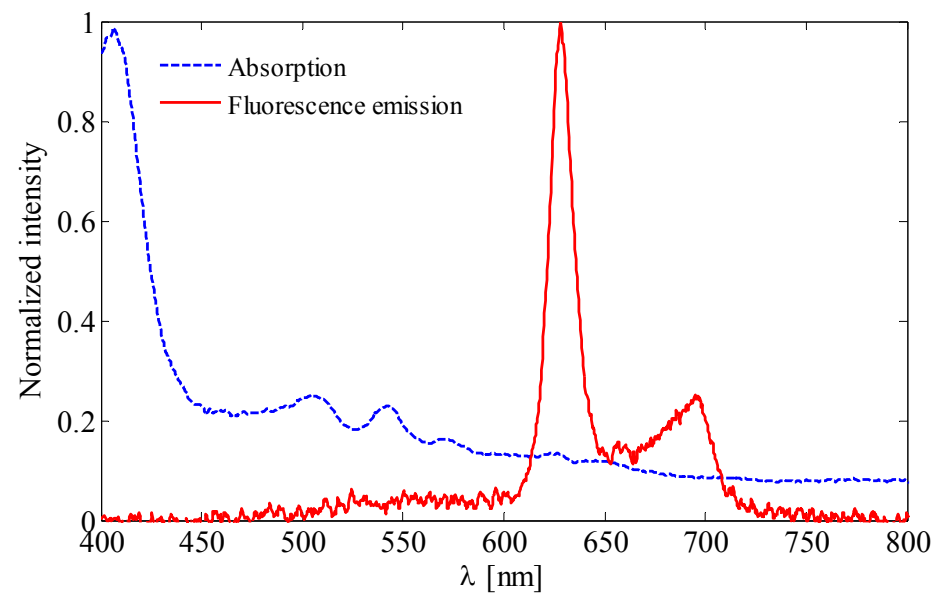

Figure 2: Absorption and emission spectra of PpIX at concentration of $14 \mathrm{mgl}^{-1}$ dissolved in DMSO.

\subsection{Fluorescence spectra and quantification}

Fluorescence signals collected from the phantoms with $\mathrm{c}_{\mathrm{PpIX}}$ of 2 and $18 \mathrm{mgl}^{-1}$ (the lowest and highest concentration used), excited with two different powers of 5 and $10 \mathrm{~mW}$ are illustrated in Figure 3. The phantoms showed comparable spectra with those collected from the brain tumor.

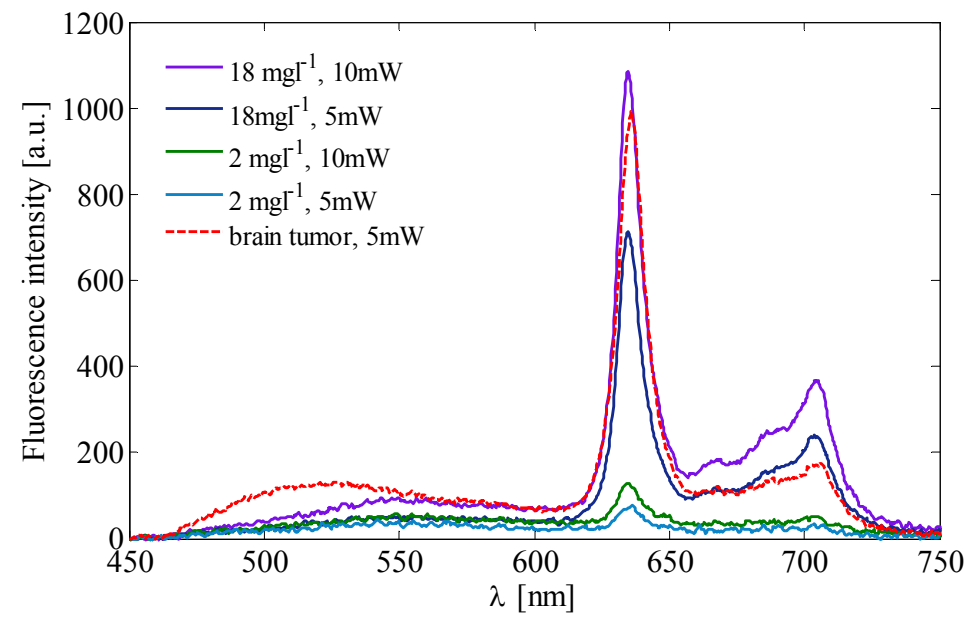

Figure 3: Fluorescence spectra collected on the phantoms with $\mathrm{c}_{\mathrm{PpIX}}$ of 2 and $18 \mathrm{mgl}^{-1}$ and one example from brain tissue. All of the above spectra are collected with $0.4 \mathrm{~s}$ integration time. 


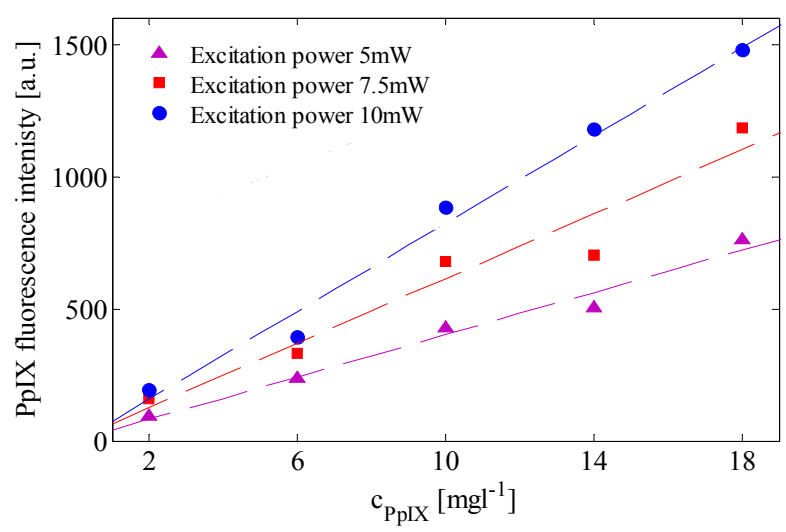

(a)

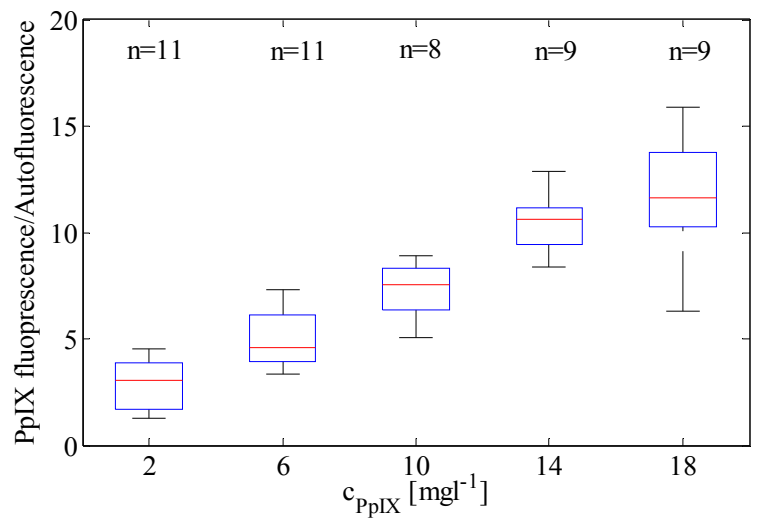

(b)

Figure 4: PpIX fluorescence intensity as a function of its concentration (a) and ratio of PpIX fluorescence at $634 \mathrm{~nm}$ by maximum emission of autofluorescence measured on the optical phantom (b).

Figure 4 (a) shows correlation $\left(\mathrm{R}^{2}>0.98, \mathrm{p}<0.01\right)$ of PpIX fluorescence intensity with PpIX concentration using the same excitation settings. For measurements performed with various laser powers and integration times, ratio of PpIX fluorescence over autofluorescence showed a statistically significant difference $(p<0.001)$ among different concentrations. Regression analysis showed a linear correlation between the PpIX concentration and the fluorescence ratio $\left(\mathrm{R}^{2}=0.79, \mathrm{p}<0.001\right)$. The linearity was lower when PpIX fluorescence alone was correlated to the PpIX concentration $\left(\mathrm{R}^{2}=0.67, \mathrm{p}<0.001\right)$.

\subsection{Photobleaching}

Decay of fluorescence (photobleaching) in phantoms with different concentrations is shown in Figure 5. An example of photobleaching in brain tumor is shown by the measured points and the curve fitting. Figure 6 shows photobleaching in terms of the time at which only $37 \%$ of the initial fluorescence is left $\left(\mathrm{t}_{37 \%}\right)$.

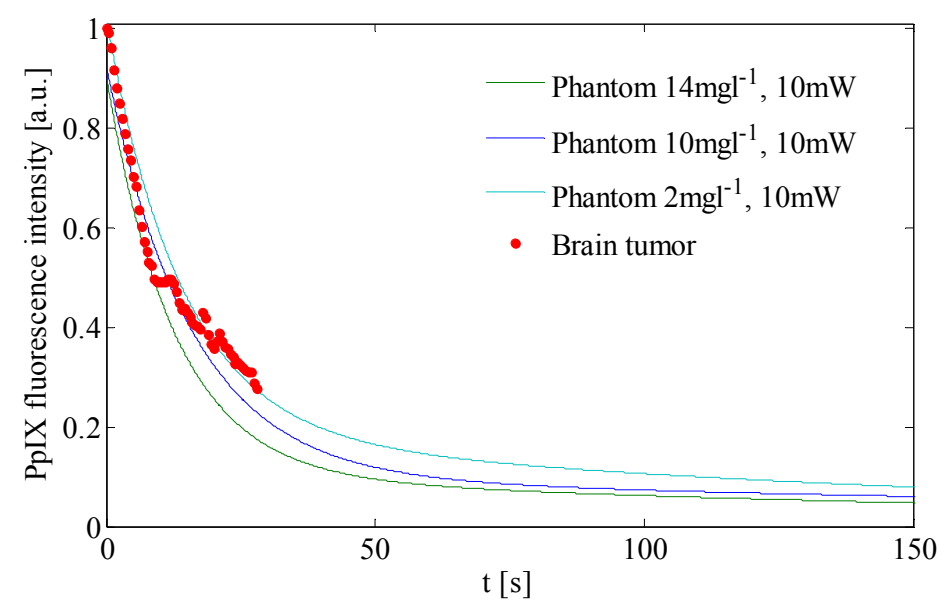

Figure 5: Photobleaching of PpIX fluorescence in phantoms with PpIX concentrations of 2, 10 and $14 \mu \mathrm{M}$. An example of fluorescence decay measurement points (dots) and curve-fit (dashed line) from brain tumor is added. 


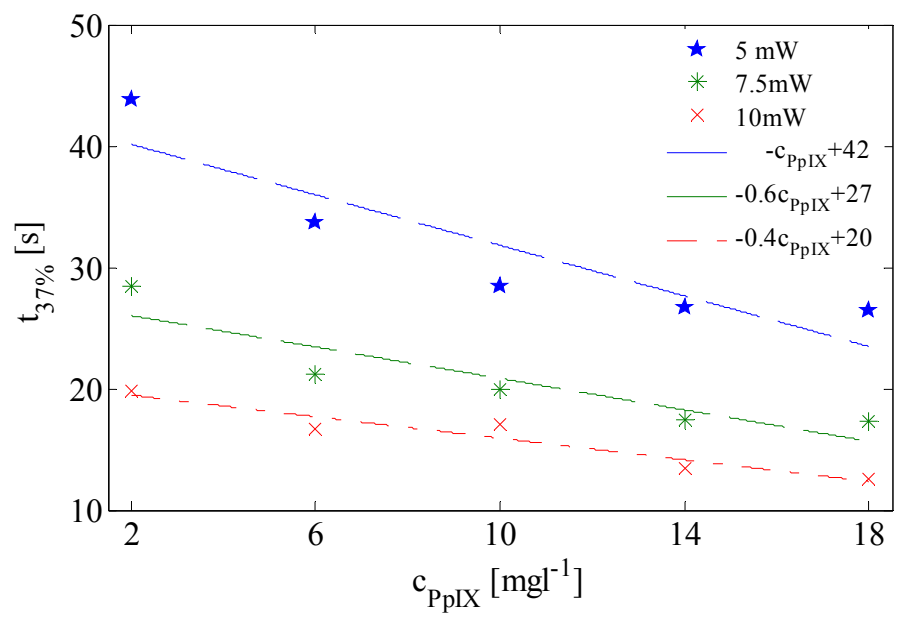

Figure 6 Photobleaching affected by the excitation power and PpIX concentration. The y axis shows the time at which only $37 \%$ of the initial fluorescence is left.

\section{DISCUSSION AND CONCLUSION}

A preliminary optical phantom model that mimicked brain tumor was characterized and compared to the data collected on the brain tumor $[4,5]$. The phantom experiments (Figure 4) showed that the PpIX fluorescence was highly correlated to the PpIX concentration $\left(\mathrm{R}^{2}>0.98\right)$ but when excitation settings were varied ratio of PpIX fluorescence over autofluorescence showed a higher correlation to the PpIX concentration than to the PpIX fluorescence. The linearity of the PpIX fluorescence and concentration has also been reported by Johansson et al $\left(R^{2}=0.99\right)$ and $\mathrm{Kim}$ et al $\left(\mathrm{R}^{2}=0.64\right)$ $[11,16]$.

Autofluorescence in brain tumor is emitted from within the tissue sampling volume. This might be more appropriately modelled in the future phantoms by adding corresponding fluorophores to the phantom. Effect of chemical environment of the phantom, although known to influence the PpIX emission [21], was not investigated; however, the emission peak of PpIX in the phantom located at $634 \mathrm{~nm}$ which is $1 \mathrm{~nm}$ blue-shifted from what is measured in the brain tumor, indicated a similar chemical environment in that of brain tumor. PpIX in DMSO solution alone had its emission peak at $627 \mathrm{~nm}$.

Mean and standard deviation of $\mathrm{t}_{37 \%}$ measured on the brain tumor was $42 \pm 24[\mathrm{~s}]$ and $26 \pm 5[\mathrm{~s}]$ for excitation powers of 5 and $10 \mathrm{~mW}$, respectively [22]. It is therefore concluded that the realized phantom with identical mechanical properties to that of brain $(0.6 \%$ agarose gel) offers a platform comparable to brain tumor for photobleaching measurements. Photobleaching measurements were not possible in the liquid phantom due to the molecular kinetics. It was however not tested whether higher or lower gel consistencies would lead to different photobleaching results.

In conclusion, the phantom provided a brain-tissue-comparable platform for investigating PpIX fluorescence and photobleaching behavior in relation to its concentration. In the phantoms, the ratio of PpIX fluorescence over autofluorescence showed a higher correlation than the PpIX fluorescence alone using different excitation light settings. Photobleaching of the PpIX was affected by the PpIX concentration where a higher concentration resulted in a faster photobleaching.

\section{ACKNOWLEDGEMENTS}

The authors would like to thank the neurosurgeons and the surgical staff at the Department of Neurosurgery, Linköping University Hospital, County Council of Östergötland for the clinical measurements and Marcus Larsson, Department of Biomedical Engineering, Linköping University, for consultation on the SCT measurements. The study was supported by the Swedish Research Council (VR) and NovaMedTech. 


\section{REFERENCES}

[1] Novotny, A., and Stummer, W., "5-Aminolevulinic Acid and the Blood-Brain Barrier - A Review," Medical Laser Application, 18(1), 36 (2003).

[2] Collaud, S., Juzeniene, A., Moan, J. et al., "On the Selectivity of 5-Aminolevulinic Acid-Induced Protoporphyrin IX Formation," Current Medicinal Chemistry - Anti-Cancer Agents, 4(3), 301-316 (2004).

[3] Stummer, W., Pichlmeier, U., Meinel, T. et al., "Fluorescence-guided surgery with 5-aminolevulinic acid for resection of malignant glioma: a randomised controlled multicentre phase III trial," The Lancet Oncology, 7(5), 392 (2006).

[4] Haj-Hosseini, N., Richter, J., Andersson-Engels, S. et al., "Optical touch pointer for fluorescence guided glioblastoma resection using 5-aminolevulinic acid," Lasers in Surgery and Medicine, 42(1), 9-14 (2010).

[5] Richter, J., Haj-Hosseini, N., Andersson-Engels, S. et al., "Fluorescence spectroscopy measurement in ultrasonic navigated resection of malignant brain tumors," Lasers in Surgery and Medicine, 43(1), 8-14 (2011).

[6] Valdés, P. A., Leblond, F., Kim, A. et al., "Quantitative fluorescence in intracranial tumor: implications for ALA-induced PpIX as an intraoperative biomarker," Journal of neurosurgery, 1-7 (2011).

[7] Ishihara, R., Katayama, Y., Watanabe, T. et al., "Quantitative Spectroscopic Analysis of 5-Aminolevulinic Acid-Induced Protoporphyrin IX Fluorescence Intensity in Diffusely Infiltrating Astrocytomas," Neurologia medico-chirurgica, 47(2), 53-57 (2007).

[8] Stummer, W., Tonn, J.-C., Goetz, C. et al., "5-ALA-Derived Tumor Fluorescence: The Diagnostic Accuracy of Visible Fluorescence Qualities as Corroborated by Spectrometry and Histology and Post-Operative Imaging," Neurosurgery, Publish Ahead of Print (2013).

[9] Bradley, R. S., and Thorniley, M. S., "A review of attenuation correction techniques for tissue fluorescence," Journal of The Royal Society Interface, 3(6), 1-13 (2006).

[10] Finlay, J. C., and Foster, T. H., "Recovery of hemoglobin oxygen saturation and intrinsic fluorescence with a forward-adjoint model,” Appl. Opt., 44(10), 1917 (2005).

[11] Kim, A., Khurana, M., Moriyama, Y. et al., "Quantification of in vivo fluorescence decoupled from the effects of tissue optical properties using fiber-optic spectroscopy measurements," Journal of Biomedical Optics, 15(6), 067006 (2010).

[12] Aalders, M. C. G., Sterenborg, H. J. C. M., Stewart, F. A. et al., "Photodetection with 5-Aminolevulinic Acidinduced Protoporphyrin IX in the Rat Abdominal Cavity: Drug-dose-dependent Fluorescence Kinetics $\mid$," Photochemistry and Photobiology, 72(4), 521-525 (2000).

[13] Gebhart, S. C., Lin, W. C., and Mahadevan-Jansen, A., "In vitro determination of normal and neoplastic human brain tissue optical properties using inverse adding-doubling ” Phys. Med. Biol., 51(8), 2011-27 (2006).

[14] Schwarzmaier, H.-J., Eickmeyer, F., Fiedler, V. U. et al., "Basic Principles of Laser Induced Interstitial Thermotherapy in Brain Tumors," Medical Laser Application, 17(2), 147-158 (2002).

[15] Yaroslavsky, A. N., Schulze, P. C., Yaroslavsky, I. V. et al., "Optical properties of selected native and coagulated human brain tissues in vitro in the visible and near infrared spectral range," Physics in medicine and biology, 47(12), 2059-2073 (2002).

[16] Johansson, A., Palte, G., Schnell, O. et al., "5-Aminolevulinic Acid-induced Protoporphyrin IX Levels in Tissue of Human Malignant Brain Tumors," Photochemistry and Photobiology, 86(6), 1373-1378 (2010).

[17] Hall, T. J., Bilgen, M., Insana, M. F. et al., "Phantom materials for elastography," Ultrasonics, Ferroelectrics and Frequency Control, IEEE Transactions on, 44(6), 1355-1365 (1997).

[18] McCracken, P. J., Manduca, A., Felmlee, J. et al., "Mechanical transient-based magnetic resonance elastography," Magnetic Resonance in Medicine, 53(3), 628-639 (2005).

[19] Lindbergh, T., Fredriksson, I., Larsson, M. et al., "Spectral determination of a two-parametric phase function forpolydispersive scattering liquids," Optics Express, 17(3), 1610-1621 (2009).

[20] Kistler, B., [Development of a tissue-like protoporphyrin IX fluorescence phantom and performance of characterisation experiments] Linköping University, (2013).

[21] Lovell, J. F., Liu, T. W. B., Chen, J. et al., “Activatable Photosensitizers for Imaging and Therapy,” Chemical Reviews, 110(5), 2839-2857 (2010).

[22] Haj-Hosseini, N., Richter, J., Andersson-Engels, S. et al., "Photobleaching behavior of protoporphyrin IX during 5-aminolevulinic acid marked glioblastoma detection." Proc. SPIE 7161, 716131-8. 